VALUTAZIONE DELL'ESPOSIZIONE ALIMENTARE A CONTAMINANTI PRESENTI SU BIOTI EDULI (PESCI, MOLLUSCHI BIVALVI E CROSTACEI) IN UN AMBIENTE COSTIERO ANTISTANTE UN'AREA MINERARIA ED INDUSTRIALE DELLA SARDEGNA (SULCIS-IGLESIENTE)

\title{
EXPOSURE ASSESSMENT TO FOOD CONTAMINANTS ON EDIBLE FISH, CLAMS AND CRUSTACEANS IN A COASTAL ENVIRONMENT FACING A MINING AND INDUSTRIAL AREA IN SARDINIA (SULCIS-IGLESIENTE)
}

\author{
Piras P.L. ${ }^{1}$, Mulas G.F. ${ }^{2}$ \\ ${ }^{1}$ Servizio Veterinario di Igiene degli Alimenti di Origine Animale dell'Azienda USL 7 di Carbonia \\ ${ }^{2}$ Servizio Ambiente del Comune di Portoscuso (con finanziamento dell'Assessorato Difesa Ambiente \\ della Regione Sardegna per il Piano di Disinquinamento del Sulcis-Iglesiente)
}

SUMMARY

KEYWORDS

\begin{abstract}
The risk assessment following exposure of the local population to the consumption of fishery products caught in the Boi Cerbus lagoon, located in country of Portoscuso (Sardinia), followed the phase of sampling, analysis and data-collection "site-specific". Overall, the fishery products samples are part of the following zoological groups: fish (grey mullets, soles and gobies), bivalve (cockles) and crustaceans (crabs). It was also agreed that further investigation were implemented in a targeted way, directing attention to critical issues that emerged from the first phase, represented by heavy metals such as Cadmium, Lead, Mercury and the (metalloid) Arsenic that, for the purposes of food safety, are still contaminants with high concern for the potential impact on human health. For the risk analysis through food intake, three different procedures for exposure assessment were considered: the regulation by law, the assessment according to US-EPA approach and the assessment according to EU harmonized method. For a wider opportunity for exposure assessment through ingestion of food from contaminated areas, also for their risk management, a single approach to the risk analysis is not be considered sufficient.
\end{abstract}

food-safety, heavy metals such as marine pollutants, dietary exposure assessment.

\section{INTRODUZIONE}

Il Comune di Portoscuso ha da tempo avviato, in accordo con obiettivi di bonifica a più ampio raggio promossi dalla Regione Sardegna, un programma di monitoraggio in una delle aree, già storicamente connotate come estrattivominerarie ed industriale-metallurgiche, che sono considerate perciò a maggior rischio per contaminazioni da metalli pesanti: il SulcisIglesiente. Relativamente alla valutazione del rischio sanitario in ambiente agricolo, cioè sui suoli e sui prodotti ortofrutticoli lì coltivati, l'area di indagine è già stata valutata attraverso uno studio svolto su mandato del Comune di Portoscuso dal Reparto Suolo e Salute del Dipartimento Ambiente e Connessa Prevenzione Primaria dell'Istituto Superiore di Sanità, tramite il monitoraggio per campionamento di diverse matrici vegetali (comprese le essenze spontanee) e valutando anche i dati forniti alla ASL 7 di Carbonia dall'Agenzia Regionale per la Protezione dell'Ambiente della Sardegna (ARPAS). Nel presente studio, la laguna di Boi Cer- 
bus (e relativa peschiera) è stata invece individuata come sito strategico per valutare anche le contaminazioni su bioti eduli, quali: pesci, molluschi bivalvi e crostacei, utilizzati come specifici bio-indicatori del comparto ambientale marino e delle acque di transizione. Nell'ambito del piano di caratterizzazione e bio-monitoraggio è stata pertanto sviluppata anche una valutazione del rischio sanitario potenziale conseguente all'esposizione a microinquinanti metallici per consumo di prodotti ittici pescati/raccolti nella laguna di Boi Cerbus. Lo studio ha fatto quindi seguito ad una estesa fase di campionamento, analisi e raccolta-dati "sito-specifici", progettata ad hoc e che ha interessato matrici ambientali (sedimenti, acque) e prodotti della pesca, come definito nel diagramma di flusso del modello concettuale riportato nella figura 1.

Figura 1. Modello concettuale per la valutazione dell'esposizione alimentare ai contaminanti presenti in laguna.

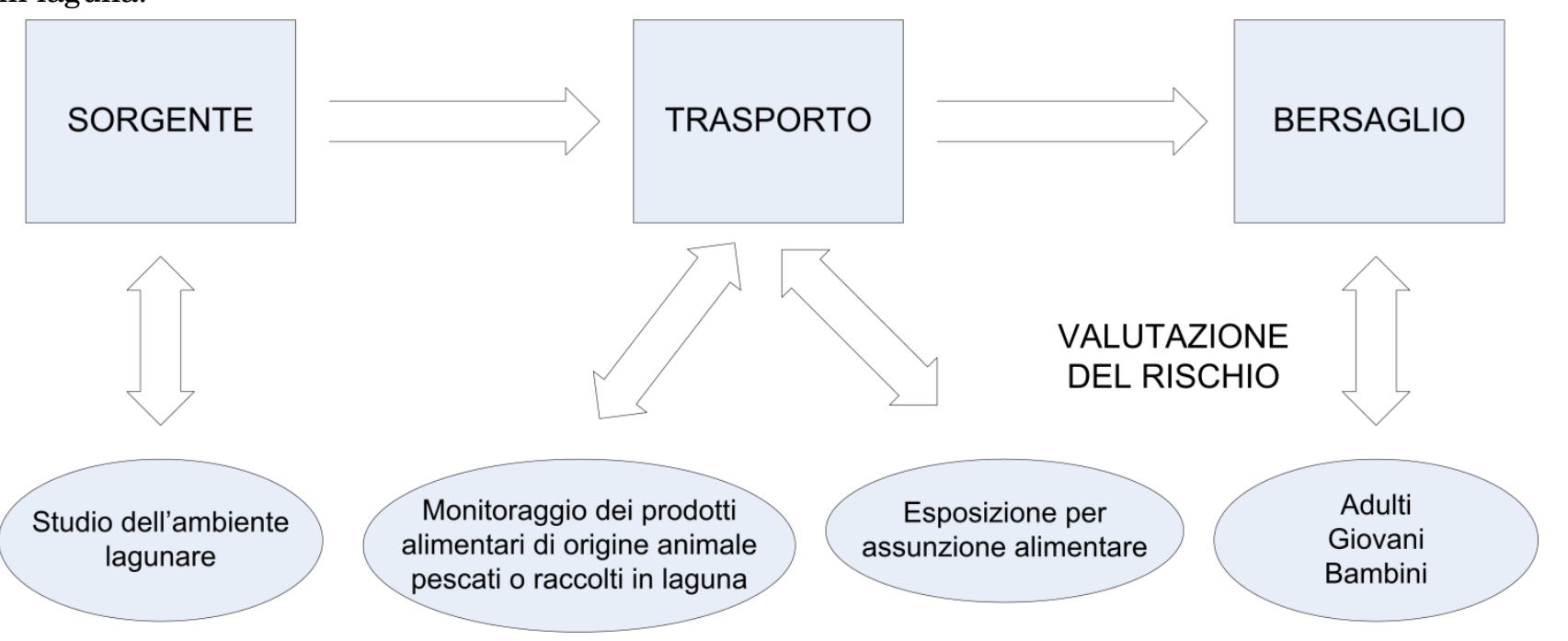

La prima parte dello studio, condotta nella stagione estiva 2008, era finalizzata alla caratterizzazione del rischio sito-specifico ed ha prodotto risultati già oggetto di un primo contributo pratico presentato ad un Convegno Nazionale AIVI (1). In tale prima fase, l'attenzione è stata posta sull'appropriato monitoraggio dei sedimenti e dei corpi idrici, oltre che delle matrici animali (bioti indicatori). Il protocollo è stato replicato nel 2010 e rivisitato (con ampliamento delle specie di bioti oggetto di indagine) anche alla luce dei risultati ottenuti nella prima fase. Sempre sulla scorta delle evidenze risultanti dalle analisi della prima fase dello studio e, particolarmente, in relazione al tenore relativo di contaminanti rilevato per ciascuna categoria dei medesimi, si è deciso inoltre di proseguire l'indagine in modo mirato, focalizzando cioè l'attenzione sulle criticità emerse, rappresentate dai microinquinanti metallici quali: l'Arsenico (As), il Cadmio (Cd), il Piombo $(\mathrm{Pb})$ ed il Mercurio (Hg), che da un punto di vista sanitario rappresentano gli elementi "in traccia" che destano particolare attenzione per il potenziale impatto sulla salute umana. Si consideri che, a tal proposito, nel 2002-2003 l'UE ha infatti costituito, nel quadro della Cooperazione Scientifica, la Task 3.2.11 su "Assessment of the dietary exposure to arsenic, cadmium, lead and mercury of the population of the EU Member States" (2).

Nello specifico, come aveva già fatto notare an- che il Joint FAO/WHO Expert Committee on Food Additives (JECFA) and Contaminants, l'As è spesso presente nei prodotti ittici anche ad elevati livelli di concentrazione, benché esso si trovi principalmente sotto forma di composti organici, decisamente meno tossici dell'As inorganico (3), in accordo con le valutazioni (4) degli esperti francesi sugli aspetti scientifici dell'inquinamento marino (IFREMer) e con la recente Opinione scientifica (5) del Panel on Contaminants in the Food Chain dell'EFSA (CONTAM). Su problematiche analoghe legate al Cd si è espressa sia il JECFA (6), sia il CONTAM in diverse occasioni (7, 8 e 9), raccomandando maggiori sforzi per ridurre l'esposizione al Cd attraverso la dieta, poiché per l'uomo gli alimenti rappresentano la fonte principale dell'Intake di tale elemento. Il $\mathrm{Pb}$ è generalmente presente a basse concentrazioni negli alimenti (10), mentre nell'epatopancreas dei prodotti ittici può essere contenuto a livelli significativi (11); inoltre, anche per il CONTAM (12), gli alimenti prodotti e lavorati in aree contaminate rappresentano la principale fonte per l'ingestione di $\mathrm{Pb}$ con la dieta. Relativamente all'assunzione alimentare del $\mathrm{Hg}$, come è noto, sono principalmente implicati i prodotti ittici (13) ed alcuni in particolare. Il metilmercurio, la principale forma in cui il mercurio è presente nei pesci, è la più tossica tra le specie del mercurio. Il contenuto di metilmercurio nei prodotti 
ittici varia, ma si assume che generalmente, oltre il $90 \%$ del mercurio totale sia presente sottoforma di metilmercurio (14).

\section{MATERIALI E METODI}

I prodotti ittici campionati erano costituiti da: teleostei appartenenti alla famiglie mugilidi (specie Mugil cephalus, Liza ramada e Liza aurata), soleidi (Solea vulgaris) e gobidi (Zosterisessor ophiocephalus), molluschi bivalvi ("cuori" della specie Cerastoderma glaucum) e crostacei brachiuri (granchi della specie Carcinus æstuarii). Sono stati esaminati complessivamente 41 pool o "campioni globali" originati da "campioni elementari" rappresentativi di ogni singola calata di pesca/raccolta e precisamente: n. 20 pool di solo muscolo (o di corpo intero nel caso dei gobidi) dei pesci considerati (provenienti dal raggio di cala di 7 punti georeferenziati), n. 15 pool di parte edibile di bivalvi "cuori" (raccolti da 5 punti georeferenziati) e n. 6 pool di granchi, escluse le "carni scure" (raccolti da 2 punti georeferenziati). Le analisi sono state eseguite dal laboratorio R\&C Lab S.r.l. (http://www.rclabsrl.it), accreditato Uni Cei En 17025 e con prove accreditate riferite ai seguenti metodi standard: EPA (Enviromental Protection Agency) 6020A/2007 con tecnica ICP-MS (Inductively Coupled Plasma-Mass Spectrometry) per la determinazione di $\mathrm{As}, \mathrm{Cd}$ e $\mathrm{Pb}$, mentre per il $\mathrm{Hg}$ si è ricorso al metodo EPA 7473/2007 con tecnica DMA (Direct Mercury Analyzer; prod. Milestone, Inc.). Poiché la tipologia ambientale monitorata col presente studio ed i relativi prodotti della pesca campionati sono assimilabili ad una produzione primaria, è da ritenersi pienamente applicabile la legislazione alimentare che fissa i limiti nelle derrate alimentari degli analiti indagati (15). Si è quindi calcolato il valore medio delle loro concentrazioni sulle diverse matrici e, per gli analiti regolamentati $(\mathrm{Cd}, \mathrm{Pb}$ e $\mathrm{Hg})$, è stato effettuato il confronto con tale normativa; è stato però calcolato anche il valore medio per l'As (non normato nelle derrate alimentari considerate) per conoscerne comunque il relativo tenore al fine di poter comunque procedere ad una valutazione dell'esposizione. La valutazione dell'esposizione svolta per i quattro analiti investigati ha utilizzato, sia l'approccio secondo il modello statunitense (US-EPA), sia quella secondo l'approccio armonizzato europeo (EU). Si è ritenuto opportuno utilizzare e confrontare i tre approcci (cioè, in primis, quello dei limiti normati e, quindi, dell'approccio valutativo secondo i due modelli US-EPA ed EU), sia per consentire la confrontabilità della valutazione dell'esposizione con le analisi di rischio sanitario sviluppate in altri contesti (che generalmente si basano proprio sulle procedure statunitensi), sia per uniformarci a quanto definito dagli esperti EFSA per la valutazione del rischio in ambito comunitario.

\section{RISULTATI}

Relativamente all'approccio preliminare, rappresentato dal confronto dei tenori rilevati con quelli massimi consentiti, in tabella 1 sono riportati i valori medi che si ricavano ( \pm D.S.) per singolo analita e per matrice/biota (con l'indicazione quantitativa dei pool da cui sono stati estratti i campioni) ed il riferimento ai limiti legali nei prodotti considerati, se normati. Nel monitoraggio sui prodotti ittici della Laguna di Boi Cerbus le risultanze analitiche sono state restituite dal laboratorio d'analisi riferite al peso secco della matrice; i dati utilizzati per i vari approcci di valutazione dell'esposizione sono stati quindi corretti per il valore dell'umidità (cioè riportati a valori "su peso fresco"), come richiesto dal Regolamento al fine di valutare la matrice alimentare così come viene normalmente consumata.

Tabella 1. Tenori di microinquinanti metallici rilevati nei prodotti pescati/raccolti nella Laguna di Boi Cerbus.

\begin{tabular}{|c|c|c|c|c|c|c|c|c|c|c|}
\hline \multirow[b]{2}{*}{$\mathrm{mg} / \mathrm{kg}$} & \multicolumn{2}{|c|}{$\begin{array}{l}\text { specie di mugilidi } \\
\left(\text { da } n^{\circ} 15 \text { pool }\right)\end{array}$} & \multicolumn{2}{|c|}{$\begin{array}{l}\text { sogliole comuni } \\
\text { (da } n^{\circ} 2 \text { pool) }\end{array}$} & \multicolumn{2}{|c|}{$\begin{array}{c}\text { ghiozzi go } \\
\text { (da n }{ }^{\circ} 3 \text { pool) }\end{array}$} & \multicolumn{2}{|c|}{$\begin{array}{l}\text { bivalvi “Cuori” } \\
\text { (da n }{ }^{\circ} 15 \text { pool) }\end{array}$} & \multicolumn{2}{|c|}{$\begin{array}{l}\text { granchi di estuario } \\
\left(\text { da }^{\circ} 6 \text { pool }\right)\end{array}$} \\
\hline & $\begin{array}{l}\text { Valore medio } \\
\text { su peso fresco }\end{array}$ & $\mathrm{LL}^{*}$ & $\begin{array}{l}\text { Valore medio } \\
\text { su peso fresco }\end{array}$ & $\mathrm{LL}^{*}$ & $\begin{array}{l}\text { Valore medio } \\
\text { su peso fresco }\end{array}$ & $\mathrm{LL}^{*}$ & $\begin{array}{c}\text { Valore } \\
\text { medio su } \\
\text { peso fresco }\end{array}$ & $\mathrm{LL}^{*}$ & $\begin{array}{c}\text { Valore } \\
\text { medio su } \\
\text { peso fresco }\end{array}$ & $\mathrm{LL}^{*}$ \\
\hline As & $4,10( \pm 2,08)$ & n.n. & $5,90( \pm 0,78)$ & n.n. & $\begin{array}{c}23,41 \\
( \pm 4,28)\end{array}$ & n.n. & $\begin{array}{c}19,32 \\
( \pm 5,03)\end{array}$ & n.n. & $\begin{array}{c}29,11 \\
( \pm 3,63)\end{array}$ & n.n. \\
\hline $\mathrm{Cd}$ & $\begin{array}{c}0,013 \\
( \pm 0,001)\end{array}$ & 0,050 & $\begin{array}{c}0,012 \\
( \pm 0,000)\end{array}$ & 0,050 & $\begin{array}{c}0,012 \\
( \pm 0,000)\end{array}$ & 0,050 & $\begin{array}{c}0,44 \\
( \pm 0,13) \\
\end{array}$ & 1,0 & $\begin{array}{c}0,18 \\
( \pm 0,06) \\
\end{array}$ & 0,50 \\
\hline $\mathrm{Pb}$ & $0,12( \pm 0,10)$ & 0,30 & $0,08( \pm 0,01)$ & 0,30 & $0,11( \pm 0,02)$ & 0,30 & $\begin{array}{c}4,11 \\
( \pm 1,46)\end{array}$ & 1,5 & $\begin{array}{c}0,67 \\
( \pm 0,11)\end{array}$ & 0,50 \\
\hline $\mathrm{Hg}$ & $0,01( \pm 0,00)$ & 0,50 & $0,01( \pm 0,00)$ & 0,50 & $0,02( \pm 0,00)$ & 0,50 & $\begin{array}{c}0,02 \\
( \pm 0,01)\end{array}$ & 0,50 & $\begin{array}{c}0,02 \\
( \pm 0,00)\end{array}$ & 0,50 \\
\hline
\end{tabular}

*LL = limiti legali. 
Per il Cd il valore limite per il "muscolo di pesce" delle specie oggetto della presente indagine è stabilito al codice 3.2.5 dell'Allegato al Regolamento comunitario pertinente (15), con due apposite note in calce a tale Regolamento che indicano, nell'ordine, che per "pesce" indicato in tale categoria è "escluso il fegato di pesce»e che solo: «Qualora i pesci siano destinati ad essere consumati per intero, il tenore massimo si applica all'intero pesce». Il significato non equivoco di quest'ultima estensione si coglie meglio ricorrendo al testo in lingua francese del Regolamento, che recita testualmente: «Lorsque le poisson doit être consommé entier, la teneur maximale s'applique au poisson entier", quindi la previsione si applica ai pesci che per ragioni predeterminate (in relazione alla specie, alla taglia e quindi alle condizioni di praticità di preparazione per il consumo alimentare) "devono" per loro natura essere consumati interi, ovvero compresi di fegato (come, ad esempio, le specie relativamente minute, tipo lo $Z$. ophiocephalus o "Ghiozzo go" del presente lavoro). Si consideri che l'esclusione di tale parenchima rappresenta spesso una condizione significativa ai fini dell'analisi valutativa di diversi contaminanti; nel caso del $\mathrm{Cd}$, per esempio, dai dati aggiuntivi delle analisi svolte separatamente sull'epatopancreas dei mugilidi campionati per la presente indagine è emerso un tenore relativo dell'ordine di 100 volte superiore rispetto al solo muscolo. Per le stesse ragioni il valore limite del $\mathrm{Cd}$ nei Crostacei è stabilito al codice 3.2.9 dell'Allegato al Regolamento, con la specifica «ad eccezione delle carni scure del granchio» che funge da parenchima omologo al fegato dei pesci.

Per il $\mathrm{Pb}$ valgono le stesse considerazioni di massima, essendo d'altronde identici i richiami in norma ai relativi codici 3.1 .5 e 3.1 .6 dell'Allegato al Regolamento, salvo l'evidenziazione, a seguito dei risultati delle analisi svolte, di un tenore relativo sul fegato rispetto al solo muscolo di ordine più basso rispetto al $\mathrm{Cd}$, comunque pari ad almeno 10 volte. In sintesi, il superamento dei tenori massimi stabiliti in norma si è verificato solo in riferimento al $\mathrm{Pb}$ : in misura relativamente elevata nei molluschi bivalvi esaminati (Cuori) e, seppur con grado inferiore, anche nei granchi di estuario. I restanti valori tabulati, $\mathrm{Hg}$ compreso, sono al di sotto dei limiti previsti e quindi vanno letti come accettabili, mentre non è possibile applicare il criterio del "limite legale" di questo primo approccio all'As, in quanto allo stato attuale non è disciplinato normativamente per le matrici alimentari considerate. Tuttavia, va registrato un tenore di As significativamente dissimile tra i bioti campionati, dell'ordine di 4-
5 volte maggiore fra quelli a stretto rapporto col fondale lagunare (specie bentoniche) rispetto alle altre (totalmente o parzialmente nectoniche) a causa, verosimilmente, proprio della loro ecologia a contatto con i sedimenti, risultati decisamente più carichi di micro-inquinanti rispetto alla colonna d'acqua sovrastante.

Oltre alla semplice valutazione dei livelli di concentrazione dei contaminanti nelle matrici ittiche campionate nella Laguna di Boi Cerbus, è stata effettuata una valutazione del rischio sanitario da consumo alimentare delle medesime matrici da parte dell'uomo. Tale valutazione è stata svolta prendendo in considerazione l'esposizione per diverse fasce d'età ("Bambini" [3-9 anni], "Giovani" [10-17 anni] e "Adulti" [1864 anni]), con riferimento al "peso corporeo medio" ed ai "consumi alimentari medi" ricavabili dai dati del survey svolto sulla popolazione italiana dall'INRAN (16).

Dopo aver raccolto e selezionato i dati di monitoraggio sui prodotti ittici, la procedura di "valutazione dell'esposizione" (Exposure Assessment) secondo l'approccio US-EPA (17), può essere schematizzata come segue: a) stima dell'Intake alimentare procapite (Intake Rate = IR) e della "quantità complessiva di contaminante che viene assunta quotidianamente tramite dieta" (C x IR); b) stima della "dose media giornaliera" (Average Daily Dose = ADD); c) calcolo del rischio valutando la "dose media giornaliera" integrata con i parametri tossicologici caratteristici dei diversi microinquinanti considerati.

Relativamente all'IR, il richiamato survey svolto e pubblicato dall'INRAN (16) fornisce una specifica stima dei consumi alimentari medi della popolazione italiana nel suo complesso, cui si è soliti ricorrere anche nelle stime svolte su una popolazione locale, come nel caso in studio, intendendo tali livelli di assunzione alimentare di determinate categorie di alimenti come "valori standardizzati" (utili in particolare nei confronti tra sottopopolazioni diversamente esposte, ma assumendo per le medesime, ai precipui fini dello studio, consumi alimentari simili o sovrapponibili). Poiché le "categorie di alimenti" utilizzate nel survey sono ampie ed, in particolare $\mathrm{i}$ prodotti ittici sono raggruppati nella voce generica di "Fish and seafood, fresh and frozen", quindi senza discriminazione delle componenti quantitative di apporto specifico nella dieta di: pesci, molluschi (bivalvi) e crostacei, appare necessario scegliere il "Valore rappresentativo di concentrazione" (C), espresso in $\mathrm{mg} / \mathrm{kg}$ di sostanza umida per ogni analita, ricorrendo al calcolo di una media ponderata dei tenori rilevati, sulla base della frazione che ciascun sottocomponente della categoria "prodotti ittici" ra- 
gionevolmente rappresenta nello specifico apporto alimentare del consumatore medio. Per esempio, i molluschi bivalvi (Cuori) ed granchi di estuario rappresentano sicuramente solo una piccola parte di tutte le matrici possibilmente afferenti alla relativa ed ampia "voce alimentare" di appartenenza e, perciò, un'attribuzione sovrastimata del consumo alimentare a tali due sotto-componenti non apparirebbe essere realisticamente rappresentativa.

È necessario quindi ipotizzare proporzioni verosimili, mantenendosi comunque ragionevolmente al riparo dal rischio di una sottostima. Se si assume prudentemente che le proporzioni nel consumo locale di pesce, da un lato, e di molluschi bivalvi (Cuori) e granchi di estuario, dall'altro, possa ripartirsi nelle percentuali rispettivamente del $80 \%$ e nel complementare $20 \%$, otteniamo le seguenti stime dei "Valori rappresentativi di concentrazione" (C) espressi in $\mathrm{mg} / \mathrm{kg}$ di analita microinquinante presente nella parte edibile del mix considerato di pro- dotti ittici: $\mathrm{As}=9,81 ; \mathrm{Cd}=0,072 ; \mathrm{Pb}=0,57 ; \mathrm{Hg}$ $=0,01$.

Ai fini della valutazione dell'esposizione è necessario infine disporre, sempre dai dati di letteratura (16) e per ciascuna delle tre classi d'età considerate, sia del corrispondente peso corporeo medio di riferimento, espresso in $\mathrm{kg}$, sia dell'IR per la categoria "prodotti ittici", espresso in grammi/giorno $(\mathrm{g} / \mathrm{d})$. Si può a questo punto calcolare la quantità di contaminante assunta quotidianamente, mediante l'apporto alimentare della voce "prodotti ittici" della Laguna di Boi Cerbus, moltiplicando i "Valori rappresentativi di concentrazione" $(\mathrm{C})$ per l'Intake alimentare (IR) procapite. Nella tabella 2 è infatti riportata la stima della quantità di contaminante $(\mathrm{C} \times \mathrm{IR})$ assunta quotidianamente tramite il consumo di prodotti ittici della Laguna di Boi Cerbus, espressa in $\mathrm{mg} /$ giorno $(\mathrm{mg} / \mathrm{d})$, per ciascun analita e per ciascuna delle tre fasce d'età considerate.

Tabella 2. Stima della quantità di contaminanti assunti quotidianamente tramite la dieta per fasce d'età.

\begin{tabular}{|c|c|c|c|c|c|c|c|}
\hline & \multirow[b]{2}{*}{ C (mg/kg) } & \multicolumn{2}{|c|}{ Bambini (3-9 anni d'età) } & \multicolumn{2}{|c|}{ Giovani (10-17 anni d'età) } & \multicolumn{2}{|c|}{ Adulti (18-64 anni d'età) } \\
\hline & & IR $(g / d)$ & $C \times \operatorname{IR}(\mathrm{mg} / \mathrm{d})$ & IR $(g / d)$ & $C \times \operatorname{IR}(\mathrm{mg} / \mathrm{d})$ & IR $(g / d)$ & C x IR (mg/d) \\
\hline As & 9,81 & \multirow{4}{*}{37,2} & 0,3649 & \multirow{4}{*}{44,2} & 0,4336 & \multirow{4}{*}{39,9} & 0,3914 \\
\hline $\mathrm{Cd}$ & 0,072 & & 0,0027 & & 0,0032 & & 0,0029 \\
\hline $\mathrm{Pb}$ & 0,57 & & 0,0212 & & 0,0252 & & 0,0227 \\
\hline $\mathrm{Hg}$ & 0,01 & & 0,0004 & & 0,0004 & & 0,0004 \\
\hline
\end{tabular}

La fase successiva della valutazione dell'esposizione prevede il calcolo del "Carico della dose media giornaliera" o Average Daily Dose (ADD), che rappresenta una stima quantitativa dell'esposizione dovuta potenzialmente all'ingestione di alimenti contaminati, espressa in termini di massa di contaminante per unità di peso corporeo per giorno ( $\mathrm{mg} / \mathrm{Kg}$ p.c. / giorno), ovvero la "dose giornaliera assunta attraverso la via alimentare"; si ottiene correlando la "quantità di contaminante assunta quotidianamente tramite la dieta" (C x IR) con altri parametri di esposizione. L'ADD è stata calcolata separatamente per le tre fasce d'età per le quali è stata effettuata la valutazione dell'esposizione, secondo la formula in uso come Standard Time-Averaging da parte dell'USEPA (17):

$$
A D D=\frac{(C \times I R) \times E F \times E D}{B W \times A T}
$$

In tale formula, i restanti parametri rappresentano le seguenti variabili: EF (Exposure Frequency) indica la "Frequenza di esposizione", cioè il numero di giorni dell'anno in cui una per- sona viene a contatto con l'inquinante (esprimibile in giorni/anno); ED (Exposure Duration) indica la "Durata di esposizione" (esprimibile in anni di vita); BW (Body Weight) indica il peso corporeo in kg; AT (Averaging Time) indica il "Tempo sul quale l'esposizione è mediata" e si esprime in giorni vissuti.

Osservando nel dettaglio tali parametri, si evidenzia che, relativamente alla variabile $\mathrm{EF}$, è necessario prendere in considerazione una "Frequenza di esposizione" effettiva, o quanto più realistica possibile, per non sovrastimare il rischio oltre ogni ragionevole cautela. A tale proposito, si consideri che in pratica nessuna delle specie campionate rappresenta un stock ittico disponibile/pescabile durante tutto l'anno ed è ragionevole attribuire al parametro EF un valore (anche cautelativamente largo) di non più di 4 mesi (120 giorni/anno) poiché lo sfruttamento produttivo della laguna è assimilabile ad una produzione primaria di tipo naturale, quindi con consumo dei prodotti ivi pescati/raccolti secondo un andamento assolutamente di tipo stagionale, anche se differenziato a seconda delle specie considerate.

La "Durata di esposizione" (ED) è pari al nume- 
ro effettivo di anni nei quali la popolazione è, o è stata, esposta all'ingestione di alimenti potenzialmente contaminati: nel caso in studio sono stati utilizzati i valori estremi (64 anni per gli "Adulti", 17 anni per i "Giovani" e 9 anni per i "Bambini") ovvero, cautelativamente, il valore massimo di ciascun arco nelle fasce d'età considerate.

Per i "Pesi corporei" (BW) di riferimento sono stati utilizzati, per coerenza, i valori riportati nella richiamata letteratura scientifica (16) che: per gli "Adulti" di 18-64 anni fissa un peso corporeo medio di $69,7 \mathrm{~kg}$, per i "Giovani" di 10-17 anni fissa quello di $52,6 \mathrm{~kg}$ ed, infine, per i "Bambini" di 3-9 anni fissa un peso medio di $26,1 \mathrm{~kg}$.

Il "Tempo sul quale l'esposizione è mediata" (AT) è pari alla durata effettiva dell'esposizione espressa in giorni; quindi, il valore da utilizzare per ciascuna fascia d'età sarà semplicemente quello ottenuto dal prodotto matematico dei giorni di un anno per la durata di esposizione (365 x ED), ovvero: $n^{\circ} 23^{\circ} 360$ giorni per la classe "Adulti", $\mathrm{n}^{\circ} 6.205$ giorni per i "Giovani" e $\mathrm{n}^{\circ}$ 3285 giorni per i "Bambini".

Nell'ultima fase della procedura di valutazione dell'esposizione e calcolo del rischio, il fattore "esposizione" viene correlato con i "parametri tossicologici" al fine di stimare quantitativamente il rischio stesso. Il calcolo del rischio viene effettuato integrando la dose media giornaliera (ADD) con la Reference Dose (RfD), cioè il parametro tossicologico caratteristico dei microinquinanti considerati. Con RfD si intende la dose (concentrazione) di riferimento di sostanza tossica per la quale, in letteratura, non vengono riportati effetti avversi per l'uomo esposto alla sostanza medesima. La stima quantitativa del rischio inerente gli effetti tossici viene quindi effettuata con il rapporto ADD / RfD, dal quale otteniamo un "Indice di rischio" o HI (Hazard Index), che esprime il "valore relativo di quanto la dose media giornaliera assunta superi la dose di riferimento".

I parametri tossicologici devono necessariamente essere reperiti in Banche-Dati validate, aggiornate e riconosciute a livello internazionale e nel presente studio i valori di RfD sono stati reperiti nella banca dati ISS/ISPESL (18). Il valore di rischio così calcolato viene poi confrontato con i criteri di accettabilità del rischio stesso: per il rischio derivante da effetti tossici, il criterio di accettabilità consiste nel "non superamento del valore di RfD" (cioè $\mathrm{HI}<1$ ).

Nella tabella 3 sono riportati i valori di Average Daily Dose (ADD) suddivisa per le tre fasce d'età, il parametro tossicologico caratteristico di ciascun microinquinante ed il valore di Hazard Index (HI) calcolato, anch'esso relativo a ciascuna fascia di età. Dai riportati si rileva che, per quanto attiene alle matrici comprese nella categoria alimentare dei "prodotti ittici" considerate nella valutazione del rischio, per ogni microinquinante considerato e per ciascuna delle tre fasce d'età, non si osserva il superamento del valore $\mathrm{RfD}$ e, quindi, potremo esprimere una giudizio favorevole relativamente al rischio da esposizione valutato secondo l'approccio USEPA, ovviamente nelle condizioni sito-specifiche indagate.

Tabella 3. Average Daily Dose (ADD) suddivisa per fasce d'età, Reference Dose (RfD) per i microinquinanti considerati e calcolo dell'Hazard Index (HI) relativo.

\begin{tabular}{|c|c|c|c|c|}
\hline & Fasce d'età & $\begin{array}{l}\text { Average Daily Dose - ADD } \\
\text { (mg/kg p.c. / giorno) }\end{array}$ & $\begin{array}{l}\text { Reference Dose - RfD } \\
\text { (mg/kg p.c. / giorno) }\end{array}$ & $\begin{array}{c}\text { Hazard Index } \\
(\text { HI = ADD / RfD) }\end{array}$ \\
\hline \multirow{3}{*}{ As } & Adulti (18-64 anni) & $4,48 \mathrm{E}-5$ & \multirow{3}{*}{$3,00 \mathrm{E}-4(*)$} & 0,15 \\
\hline & Giovani (10-17 anni) & $6,58 \mathrm{E}-5$ & & 0,22 \\
\hline & Bambini (3-9 anni) & $1,12 \mathrm{E}-4$ & & 0,37 \\
\hline \multirow{3}{*}{$\mathrm{Cd}$} & Adulti (18-64 anni) & $4,23 \mathrm{E}-6$ & \multirow{3}{*}{$5,00 \mathrm{E}-4(* *)$} & 0,01 \\
\hline & Giovani (10-17 anni) & $6,21 \mathrm{E}-6$ & & 0,01 \\
\hline & Bambini (3-9 anni) & $1,05 \mathrm{E}-5$ & & 0,02 \\
\hline \multirow{3}{*}{$\mathrm{Pb}$} & Adulti (18-64 anni) & $1,62 \mathrm{E}-5$ & \multirow{3}{*}{$3,50 \mathrm{E}-3(* * *)$} & 0,005 \\
\hline & Giovani (10-17 anni) & $2,37 \mathrm{E}-5$ & & 0,007 \\
\hline & Bambini (3-9 anni) & $4,03 \mathrm{E}-5$ & & 0,012 \\
\hline \multirow{3}{*}{$\mathrm{Hg}$} & Adulti (18-64 anni) & $5,18 \mathrm{E}-8$ & \multirow{3}{*}{$3,00 \mathrm{E}-4(* * * *)$} & 0,0002 \\
\hline & Giovani (10-17 anni) & $7,61 \mathrm{E}-8$ & & 0,0003 \\
\hline & Bambini (3-9 anni) & $1,29 \mathrm{E}-7$ & & 0,0004 \\
\hline
\end{tabular}

(*) As inorganico: fonte IRIS - Integrated Risk Information System (US-EPA, last revised 1993);

(**) Cd: fonte IRIS - Integrated Risk Information System (US-EPA, last revised 1994);

(***) $\mathrm{Pb}$ : fonte "Drinking Water Guidelines" (WHO, 1993);

(****) Hg: fonte IRIS - Integrated Risk Information System (US-EPA, last revised 1995)

Sulla base della vigente disciplina comunitaria sulla sicurezza alimentare, è stata effettuata un'ulteriore valutazione dell'esposizione, per i microinquinanti considerati, secondo l'approccio 
armonizzato europeo (EU) utilizzando il parametro tossicologico Provisional Tollerable Weekly Intake (PTWI), che rappresenta la "Quantità massima di contaminante tollerabile settimanalmente" in funzione del peso corporeo dell'individuo. Per la valutazione secondo l'approccio EU, si è moltiplicato il valore di PTWI di ciascun analita per il peso corporeo ottenendo, così, la quantità settimanale tollerabile pro capite. Moltiplicando poi la quantità di contaminante assunta quotidianamente con la dieta (C x IR) per sette giorni, si ottiene il valore confrontabile con la quantità settimanale pro capite tollerabile.

Già nei "considerato" del preambolo al Regolamento comunitario che definisce i tenori massimi di alcuni contaminanti nei prodotti alimentari (15) sono richiamati i PTWI per $\mathrm{Cd}, \mathrm{Pb}$ e
$\mathrm{Hg}$ approvati a livello internazionale, nella misura rispettivamente di $7 \mathrm{\mu g} / \mathrm{kg}$ di peso corporeo (7), di $25 \mathrm{\mu g} / \mathrm{kg}$ di peso corporeo (12) e di 1,6 $\mathrm{\mu g} / \mathrm{kg}$ di peso corporeo (14), per settimana. Pur se non considerato nel predetto Regolamento comunitario, la valutazione dell'esposizione secondo l'approccio EU è stata effettuata anche per l'As, per il quale il JECFA ha stabilito un PTWI di $15 \mu \mathrm{g} / \mathrm{kg}$ di peso corporeo (5), sempre per settimana.

Nella tabella 4 sono riportati i predetti valori del PTWI espressi in funzione dei pesi corporei relativi a ciascuna delle tre fasce d'età, l'Intake pro capite stimato settimanalmente ed il valore relativo dell'Intake stesso rispetto ai valori $\mathrm{di}$ PTWI. Affinché sia giudicato accettabile, tale rapporto non deve superare, anche in questo caso, l'unità (cioè Intake/PTWI < 1).

Tabella 4. Confronto tra i valori di PTWI e gli Intake stimati per Arsenico, Cadmio, Piombo e Mercurio.

\begin{tabular}{|c|c|c|c|c|}
\hline & Fasce d'età & $\begin{array}{c}\text { PTWI x kg di peso corporeo } \\
\text { ( } \boldsymbol{\mu g} / \text { pro capite / settimana) }\end{array}$ & $\begin{array}{c}\text { Intake settimanale } \\
\text { stimato } \\
\text { ( } \mu_{\mathrm{g} / \mathrm{pro} \text { capite } / \text { settimana })}\end{array}$ & $\begin{array}{l}\text { Valore dell'Intake } \\
\text { rispetto al PTWI }\end{array}$ \\
\hline \multirow{3}{*}{ As } & Adulti (18-64 anni) & 1046 & 900 & 0,86 \\
\hline & Giovani (10-17 anni) & 789 & 998 & 1,26 \\
\hline & Bambini (3-9 anni) & 392 & 840 & 2,15 \\
\hline \multirow{3}{*}{$\mathrm{Cd}$} & Adulti (18-64 anni) & 488 & 7 & 0,01 \\
\hline & \begin{tabular}{|l} 
Giovani (10-17 anni) \\
\end{tabular} & 368 & 7 & 0,02 \\
\hline & Bambini (3-9 anni) & 183 & 6 & 0,03 \\
\hline \multirow{3}{*}{$\mathrm{Pb}$} & Adulti (18-64 anni) & $1 \cdot 743$ & 53 & 0,03 \\
\hline & \begin{tabular}{|l} 
Giovani (10-17 anni) \\
\end{tabular} & 1315 & 58 & 0,04 \\
\hline & Bambini (3-9 anni) & 653 & 49 & 0,08 \\
\hline \multirow{3}{*}{$\mathrm{Hg}$} & Adulti (18-64 anni) & 112 & 1 & 0,01 \\
\hline & \begin{tabular}{|l} 
Giovani (10-17 anni) \\
\end{tabular} & 84 & 1 & 0,01 \\
\hline & Bambini (3-9 anni) & 42 & 3 & 0,06 \\
\hline
\end{tabular}

Si rileva che il valore di PTWI è superato solamente per l'As nei "Bambini", per oltre il doppio, e nella fascia d'età "Giovani", per un $26 \%$ in più della dose accettabile. I valori dell'Intake stimati per gli altri analiti, nelle tre fasce d'età considerate sono relativamente bassi, compresi tra il valore minimo del Cd per gli "Adulti", pari all'1\% del PTWI, a quelli del Pb nei "Bambini", pari all'8\% del relativo PTWI.

\section{CONSIDERAZIONI E CONCLUSIONI}

Per l'analisi del rischio sanitario attraverso l'assunzione alimentare, sono state considerate tre diverse procedure di valutazione dell'esposizione: la valutazione secondo normativa, la valutazione secondo l'approccio US-EPA e la valutazione secondo l'approccio armonizzato EU.

Va considerato innanzitutto che la normativa disciplinante i tenori massimi sulle derrate alimentari di alcuni contaminanti non comprende tutti gli analiti indagati nel caso in studio (è in- fatti escluso l'As) e, pertanto, la valutazione dell'esposizione secondo normativa deve essere comunque integrata con altri approcci valutativi. Inoltre, riferendoci al $\mathrm{Pb}$, le matrici per le quali si riscontra una concentrazione media superiore al valore limite normato (molluschi bivalvi e granchi), assume invece un profilo di relativa "accettabilità" qualora si proceda con altri approcci, meglio ponderati (ai fini della valutazione dell'esposizione) sull'Intake alimentare effettivo e su "gruppi a rischio", come possono esserlo le diverse fasce d'età.

Si tenga inoltre presente che si è implicitamente partiti dall'assunzione (estremizzata) che il consumo locale dei prodotti ittici provenga unicamente dall'area oggetto di indagine, attribuendo quindi l'intero valore dei consumi (IR) ai prodotti alimentari monitorati. È stato invece ponderato il consumo delle sotto-categorie di prodotti ittici: relativamente alla voce "molluschi bivalvi / Cuori" e "granchi diestuario" (già valutati "critici" sotto il profilo dei limiti normati, per i tenori di $\mathrm{Pb}$ ) e a quella dei "ghiozzi go", a cui si uniscono in ragione dei relativi alti te- 
nori di As (non speciato) riscontrati, si è infatti ritenuto eccessivo attribuire loro l'intero IR della "Voce alimentare" alla quale afferiscono. Tuttavia, allo stato attuale delle informazione sitospecifiche e dall'insieme delle valutazioni effettuate, appare appropriato sotto il profilo della "Gestione del Rischio" non consentire, cautelativamente, la pesca/raccolta di molluschi bivalvi e di granchi di estuario nella laguna di Boi Cerbus e zone limitrofe, a conforto delle Ordinanze Sindacali già emanate a tal fine dalle Autorità Sanitarie Locali.

In sintesi quindi, per una più ampia possibilità di valutazione del rischio sanitario connesso all'ingestione di alimenti provenienti da aree compromesse a livello ambientale, anche ai fini della gestione del rischio considerato, non è da ritenersi sufficiente il ricorso ad un unico approccio valutativo del rischio stesso. Infatti, con il presente lavoro si è potuto evidenziare che, dalla valutazione secondo la vigente normativa, si ha il superamento dei limiti fissati per il $\mathrm{Pb}$ in alcune matrici monitorate; dalla valutazione del rischio sanitario effettuata secondo l'approccio US-EPA, con i parametri utilizzati, non si evidenzia invece rischio per nessuno degli analiti considerati; mentre con la valutazione secondo l'approccio EU si evidenzia il rischio ipotetico dovuto all'As per le fasce d'età più basse (identificabili quindi come "gruppi a rischio"). Tuttavia, relativamente a tale rischio ipotetico va rimarcata l'assoluta importanza di effettuare la speciazione chimica dei composti dell'As per ogni valutazione del rischio ad esso associato in quanto, come è noto, la sua tossicità è strettamente connessa con la forma chimica in cui tale elemento si presenta: i composti organici sono infatti generalmente molto meno tossici di quelli inorganici. Nei pesci e nei crostacei l'As si trova infatti principalmente sotto forma organica di arsobetaina, considerata virtualmente non tossica, mentre nei molluschi e nelle alghe l'As è presente principalmente come arsenozuccheri, la cui tossicità è meno conosciuta, ma si ritiene sia anch'essa ragionevolmente molto bassa.

\section{BIBLIOGRAFIA E DOCUMENTI DI RIFERIMENTO (CON URL INTERNET ATTIVI AL 15 MAGGIO 2011)}

1. Caratterizzazione del rischio in un ambiente costiero antistante un'area mineraria ed industriale della Sardegna (Sulcis-Iglesiente) tramite campionamento a cluster e ricerca di contaminanti su bioti eduli (pesci e molluschi bivalvi) - Pierluigi Piras, Gianfranco Mulas - Atti XX Convegno Nazionale
AIVI:
2010
$[7$
pp.]

(www.aivi.it/doc/47313418.xls)

2. Assessment of the dietary exposure to arsenic, cadmium, lead and mercury of the population of the EU Member States - Cooperation on questions relating to food - Report of experts participating in Task 3.2.11, March 2004 [125 pp.] (http://ec.europa.eu/food/food/chemicalsafety /contaminants/scoop_3-2-

11_heavy_metals_report_en.pdf)

3. Safety evaluation of certain food additives and contaminants - Prepared by the Thirtythird meeting of the Joint FAO/WHO Expert Committee on Food Additives (JECFA), 1989 - WHO Food Additives Series: $24-$ Arsenic:

$155-219$

http://www.inchem.org/documents/jecfa/jec mono/v024je08.htm)

4. L'arsenic en milieu marin. Biogéochimie et écotoxicologie - Pierre Michel - Institut Français de Recherche pour l'Exploitation de la Mer (I.F.R.E.Mer) - Reperes Ocean (4) 1993 [65 pp.] (http://archimer.ifremer.fr/doc/1993/rapport1448.pdf)

5. Scientific Opinion on Arsenic in Food - EFSA Panel on Contaminants in the Food Chain [CONTAM] (EFSA-Q-2008-425) - European Food Safety Authority (EFSA) Journal 2009; 7(10): $1351 \quad$ [199 pp.] (http://www.efsa.europa.eu/it/efsajournal/do c/1351.pdf)

6. Safety evaluation of certain food additives and contaminants - Prepared by the Sixtyfirst meeting of the Joint FAO/WHO Expert Committee on Food Additives (JECFA), 2004 - WHO Food Additives Series: 52 Cadmium: 505-563

(http://whqlibdoc.who.int/publications/2004/ 924166052x.pdf)

7. Cadmium in food - Scientific Opinion of the Panel on Contaminants in the Food Chain [CONTAM] (EFSA-Q-2007-138) - European Food Safety Authority (EFSA) Journal 2009; $980, \quad 1-139 \quad-$ (http://www.efsa.europa.eu/it/efsajournal/do c/980.pdf)

8. Statement on tolerable weekly intake for cadmium - Statement of the CONTAM Panel (EFSA-Q-2010-01008) - European Food Safety Authority (EFSA) Journal 2011; 9(2): 1975 [19 pp.] (http://www.efsa.europa.eu/it/efsajournal/do c/1975.pdf)

9. Comparison of the Approaches Taken by EFSA and JECFA to Establish a HBGV for Cadmium - Scientific Report of EFSA (EFSA-Q-2010-01135) - EFSA Journal 2011; 
9(2): $2006 \quad\left[\begin{array}{ll}28 & \text { pp. }] \quad-\end{array}\right.$ (http://www.efsa.europa.eu/it/efsajournal/do c/2006.pdf)

10. Safety evaluation of certain food additives and contaminants - Prepared by the Fiftythird meeting of the Joint FAO/WHO Expert Committee on Food Additives (JECFA), 2000 - WHO Food Additives Series: 44 Lead: $\quad$ 965-986

(http://www.inchem.org/documents/jecfa/jec mono/v44jec12.htm)

11. Le plomb en milieu marin. Biogéochimie et écotoxicologie - Daniel Cossa, Françoise Elbaz-Poul., Mauricette Gnassia-Barelli et Michèle Romeo - Institut Français de Recherche pour l'Exploitation de la Mer (I.F.R.E.Mer) - Reperes Ocean (3) 1993 [77 pp.]

(http://archimer.ifremer.fr/doc/1993/rapport1449.pdf)

12. Scientific Opinion on Lead in Food - EFSA Panel on Contaminants in the Food Chain [CONTAM] (EFSA-Q-2007-137) - European Food Safety Authority (EFSA) Journal 2010; 8(4): $1570 \quad$ [147 $\quad$ pp.] (http://www.efsa.europa.eu/it/efsajournal/do c/1570.pdf)

13. Safety evaluation of certain food additives and contaminants - Prepared by the Sixtyfirst meeting of the Joint FAO/WHO Expert Committee on Food Additives (JECFA), 2004 - WHO Food Additives Series: 52 Methylmercury : 565-623 (http://whqlibdoc.who.int/publications/2004/ 924166052x.pdf)

14. Opinion of the Scientific Panel on contaminants in the food chain [CONTAM] related to mercury and methylmercury in food (EFSA-Q-2003-030) - European Food Safety Authority (EFSA) Journal 2004; 34, 1-14 (http://www.efsa.europa.eu/it/efsajournal/do c/34.pdf)
15. Regolamento (CE) n. 1881/2006 [della Commissione] del 19 dicembre 2006 che definisce i tenori massimi di alcuni contaminanti nei prodotti alimentari - (G.U.C.E. 20/12/2006, L 364: 5-24) modificato dal Regolamento (CE) n. 629/2008 (G.U.C.E. 03/07/2008, L 137: 6-9) - testo consolidato (http://eur-

lex.europa.eu/LexUriServ/LexUriServ.do?ur i=CONSLEG:2006R1881:20100701:IT:PDF)

16. The Italian National Food Consumption Survey INRAN-SCAI 2005-06: main results in terms of food consumption - Catherine Leclercq, Davide Arcella, Raffaela Piccinelli, Stefania Sette, Cinzia Le Donne and Aida Turrini - Public Health Nutrition, 12(12) 2009: 2504-2532

(http://journals.cambridge.org/action/display Fulltext?type $=1 \&$ fid $=6574248 \&$ jid $=$ PHN\&v olumeId=12\&issueId=12\&aid=6574240\&bo dy-

Id=\&membershipNumber=\&societyETOCS ession)

17. Risk Assessment Guidance for Superfund: Volume III - Part A, Process for Conducting Probabilistic Risk Assessment - Appendix D - Advanced Modeling Approaches for Characterizing Variability and Uncertainty - Office of Emergency and Remedial Response U.S. EPA 540-R-02-002, December 2001 [27 pp.]

(http://www.epa.gov/oswer/riskassessment/r ags3adt/pdf/appendixd.pdf)

18. APAT (Agenzia per la Protezione dell'Ambiente e per i Servizi Tecnici) - Criteri metodologici per l'applicazione dell'analisi assoluta di rischio ai siti contaminati - Appendice O (Rev. 2 - Marzo 2008): 11-16 (http://www.isprambiente.gov.it/site/_files/te mi/Banca_dati_ISS_ISPESL_Maggio_2009.x ls) 